\title{
Perceptual grouping in change detection
}

\author{
YUHONG JIANG \\ Massachusetts Institute of Technology, Cambridge, Massachusetts \\ MARVIN M. CHUN \\ Yale University, New Haven, Connecticut \\ and \\ INGRID R. OLSON \\ University of Pennsylvania, Philadelphia, Pennsylvania
}

\begin{abstract}
Detection of an item's changing of its location from one instance to another is typically unaffected by changes in the shape or color of contextual items. However, we demonstrate here that such location change detection is severely impaired if the elongated axes of contextual items change orientation, even though individual locations remain constant and even though the orientation was irrelevant to the task. Changing the orientations of the elongated stimuli altered the perceptual organization of the display, which had an important influence on change detection. In detecting location changes, subjects were unable to ignore changes in orientation unless additional, invariant grouping cues were provided or unless the items changing orientation could be actively ignored using feature-based attention (color cues). Our results suggest that some relational grouping cues are represented in change detection even when they are task irrelevant.
\end{abstract}

Change detection has been a useful tool with which to study visual attention and short-term memory in visual perception (Rensink, 2002; for a nice collection of studies, see Visual Cognition, 7, 2000). It creates surprising but convincing demonstrations of how visual representations are impoverished (Simons \& Levin, 1997) and also how such representations can be enhanced by focal attention (Rensink, O'Regan, \& Clark, 1997). It has become a standard paradigm for studying properties of visual short-term memory (VSTM; Luck \& Vogel, 1997; Pashler, 1988; Phillips, 1974). Most studies have focused on change detection of a collection of individual items, with an interest in how each item is represented and how its change is detected.

Change detection can also be used to probe the nature of relational encoding among items: how items are perceptually grouped and how such grouping is carried from perception to short-term memory. One approach is to cue attention to an element within a perceptual group, defined by proximity or connectedness, and then test the success with which changes of items in the same or a different group are detected (Woodman, Vecera, \& Luck, 2003). Same-group items are detected better than different-

We thank Mike Mangini, Lawrence E. Marks, Robert Melara, Ron Rensink, and Rebecca Saxe for valuable comments and criticisms. This research is supported in part by a Helen Hay Whitney research fellowship to Y.J. Correspondence should be sent to Y. Jiang, who is now at the Department of Psychology, Harvard University, 33 Kirkland Street, Room 820, Cambridge, MA 02138 (e-mail: yuhong@wjh.harvard .edu). group items are, suggesting that perceptual grouping is preserved in transferring information to short-term memory. Another approach relies on the principle of encoding specificity (Tulving, 1974) and varies the retrieval context so that, across frames of changes, the context surrounding the critical change either matches or mismatches the context during encoding (Jiang, Olson, \& Chun, 2000). Using the latter approach, we found that the detection of location changes is unaffected by changes in the color or shape of the contextual items but is disrupted by changes in the locations of the surrounding items. This suggests that the spatial relation formed by adjacent items is encoded and represented in VSTM, but surface features are not.

In this study, we further pursued the representation of perceptual grouping in change detection tasks. What role does perceptual grouping play in the detection of changes in item locations? Our own research in the past has suggested that perceptual grouping plays a significant role if it is relevant to the task but can be largely ignored if it is irrelevant to the task. If, for example, subjects are asked to remember the locations of chromatic items and to ignore the locations of achromatic items, then change detection is completely determined by changes of the chromatic items and is unaffected by changes of the achromatic items (Jiang et al., 2000). In this case, color grouping is relevant, and hence the target group (chromatic items) is attended and transferred into VSTM. If, however, color grouping is irrelevant, it is largely ignored. For instance, consider a set of red and green items forming two color groups - red items and green items. The perceptual orga- 
nization (grouping) is preserved when these items maintain their colors across frames but is changed if some of the red items swap colors with some of the green items. Nonetheless, if subjects are asked to remember the locations of all the items, then location change detection is unaffected by such color swapping (Jiang et al., 2000). Thus, it appears that if color grouping is relevant to the task, then the target group is effectively selected and stored in VSTM; but if it is irrelevant to the task, then change detection of locations can proceed independently of changes in the perceptual organization of the display.

The purpose of our present study was to demonstrate that some types of perceptual grouping cues that are irrelevant to the task cannot be effectively ignored in change detection. We presented subjects with two dot arrays separated by a brief interstimulus interval of $1 \mathrm{sec}$, and the subjects were required to remember the locations of these dots and to judge whether the two arrays occupied identical locations or whether there was a change in one location. Using these displays, we have previously shown that change detection of dot locations was unaffected by the color change of these items (Jiang et al., 2000). In the present study, we added a short line segment interposing each dot. From the memory to the probe display, these line segments either maintained their orientation or changed orientation. A schematic sample of an orientation change is illustrated in Figure 1.

Note that in this design the locations of the dots were relevant whereas the orientations of the line segments were not. Nonetheless, elongated lines tend to group by spatial proximity. The change in their orientations produced a change in the proximity relations and, hence, in- duced changes in perceptual grouping. Can irrelevant perceptual grouping always be ignored in change detection of item locations?

\section{EXPERIMENT 1}

\section{Method}

Subjects. Ten observers were tested in Experiment 1. The subjects were recruited from Yale University, Vanderbilt University, and Harvard University. Their ages ranged from 18 to 30 years. All the subjects signed an informed consent form before the test and were fully debriefed after the experiment. They had normal or corrected-to-normal visual acuity and normal color vision.

Procedure. Each trial started with a $500-\mathrm{msec}$ white fixation point $\left(0.3^{\circ} \times 0.3^{\circ}\right)$, which was followed by an initial display containing eight elements in randomly selected cells in an invisible $8 \times 8$ matrix that subtended $17.5^{\circ} \times 17.5^{\circ}$. Each item was positioned at the center of a cell. The initial display was presented for $400 \mathrm{msec}$, followed by a blank display of $1,000 \mathrm{msec}$. Next, the probe display, containing eight elements, was presented. Each element was a black dot $\left(0.4^{\circ} \times 0.4^{\circ}\right)$ with a white line segment $\left(1.2^{\circ} \times 0.2^{\circ}\right)$ interposed at its center. On $50 \%$ of the trials, all of the dots maintained their locations from the memory to the probe display. On the other $50 \%$ of the trials, one dot was randomly selected from the eight and was relocated to a previously unoccupied cell. The observers were required to press one of two keys to report whether they detected a location change. They were instructed that the location of an item was indicated by the black dot, and they should ignore the white line elements.

In addition to location changes, each line segment either maintained its initial randomly chosen orientation or changed its orientation by $45^{\circ}-90^{\circ}$ (with $50 \%$ probability). Each subject was tested in 12 practice and 80 experimental trials $(80=2$ location [change vs. nonchange] $\times 2$ orientation [change vs. nonchange] $\times 20$ cases).

Equipment. The experiment was programmed using MacProbe 1.88 (Hunt, 1994). The observers were tested individually in a room with normal interior lighting. They sat at an unrestricted distance

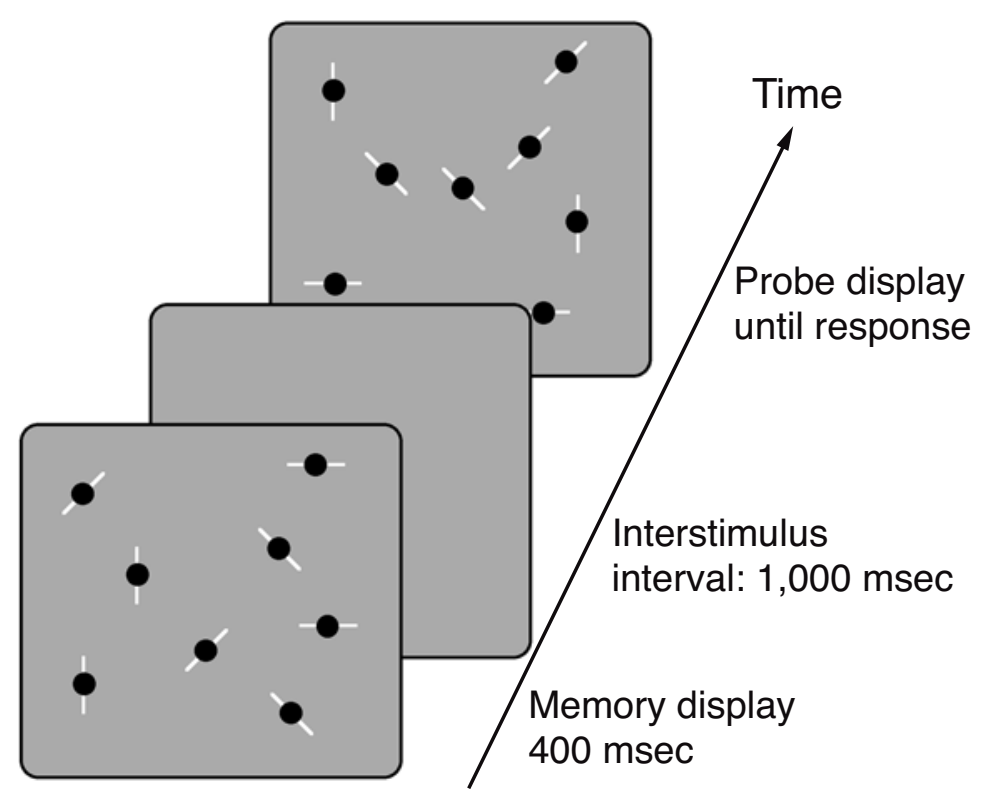

Figure 1. A schematic example of the displays used in Experiment 1. This example shows a trial in which a dot changed its location and the line segments all changed orientations. Not shown are control trials in which the line segments maintained their orientations. 
from the computer screen of about $57 \mathrm{~cm}$, at which distance $1 \mathrm{~cm}$ corresponded to $1^{\circ}$ of visual angle.

Data analysis. In this study, we calculated $A^{\prime}$ as a measure of the subject's sensitivity (Grier, 1971). We also measured percent correct and $d^{\prime}$. The overall pattern of results were the same whether $A^{\prime}$, percent correct, or $d^{\prime}$ was used. $A^{\prime}$ was preferred as a measure of memory accuracy (Donaldson, 1993).

\section{Results}

Mean $A^{\prime}$ for change detection of dot location was .91 when the orientations of the interposing lines did not change and was .76 when they did change. This difference was statistically significant $[t(9)=3.48, p<.007]$. Thus, change detection of locations was significantly impaired by orientation change of irrelevant line segments.

\section{Discussion}

In change detection of dot locations, the subjects were unable to ignore orientation change of interposing line segments. This suggests that at least some contextual mismatch between encoding and retrieval, irrelevant to the critical dimension of the task, could not be ignored.

We performed further experiments (Experiments 1B$1 \mathrm{~J})$ in order to clarify the conditions under which irrelevant orientation changes could and could not be ignored. Table 1 summarizes the results. These data ruled out some explanations of the disruption effect observed in Experiment 1. For example, (1) item rotation in general did not produce disruption in location change detection, as long as the item was not elongated (e.g., gratings and cross patterns); and (2) the amount of transient changes was not critical because the rotation of cross patterns led to as much (or more) transient change but did not disrupt performance. The fact that disruption was significant when the retention interval was only $50 \mathrm{msec}$ also suggests that the effect is tied to the perception of the stimuli and is not just a property of VSTM retention. In sum, it appears that orientation changes in the long axis of elongated objects were critical for the disruption of location change detection.

Such disruption can be accounted for by the mismatch in perceptual grouping between the memory and the probe displays. Items in an array are not represented individually but relationally. Orientation change in elongated objects altered the global organization of the items. Figure 2 illustrates how the global organization of elongated items is affected by their orientations. Whereas the lines on the left panel tend to be grouped horizontally owing to spatial proximity (Palmer, 1999), after a $90^{\circ}$ rotation, the lines on the right panel tend to be grouped vertically, even though the center locations of each item did not change.

If subjects are provided with an additional, explicit frame of reference that is constant across changes in orientation, then grouping by proximity should play a smaller role. The next experiment tests this hypothesis.

\section{EXPERIMENT 2}

Experiment 2 tested whether an added constant reference frame would reduce the disruption effect observed in Experiment 1. This additional reference frame was provided in two ways: Line stimuli were presented within a regularly spaced, visible grid (Experiment 2A), or the centers of line stimuli were connected by chromatic links

Table 1

Location Change Detection $\left(A^{\prime}\right)$ Using Various Stimuli

\begin{tabular}{|c|c|c|c|c|c|c|}
\hline Experiment & Stimuli & $\begin{array}{l}\text { Type of Item Change From } \\
\text { Memory to Probe Displays }\end{array}$ & $\begin{array}{l}A^{\prime} \text { With } \\
\text { Change }\end{array}$ & $\begin{array}{l}A^{\prime} \text { Without } \\
\text { Change }\end{array}$ & $N$ & $p$ Level \\
\hline $1 \mathrm{~B}$ & - & $\begin{array}{l}\text { Rotation; } \\
\text { ISI }=1,000 \mathrm{msec}\end{array}$ & .73 & .87 & 7 & .006 \\
\hline $1 \mathrm{C}$ & & $\begin{array}{l}\text { Rotation; } \\
\text { ISI }=50 \mathrm{msec}\end{array}$ & .83 & .98 & 7 & .002 \\
\hline $1 \mathrm{D}$ & & $\begin{array}{l}\text { Addition or deletion } \\
\text { of line segments }\end{array}$ & .81 & .88 & 8 & .005 \\
\hline $1 \mathrm{E}$ & $-1-1$ & $\begin{array}{l}\text { Length change } \\
\text { Same subjects: Rotation }\end{array}$ & $\begin{array}{l}.88 \\
.73^{*}\end{array}$ & $\begin{array}{l}.95 \\
.94\end{array}$ & 6 & $\begin{array}{l}.007 \\
.001\end{array}$ \\
\hline $1 \mathrm{~F}$ & $\Rightarrow \| \circlearrowleft$ & Rotation & .88 & .88 & 11 & $>.50$ \\
\hline $1 \mathrm{G}$ & $+x+t$ & Rotation & .86 & .88 & 10 & $>.35$ \\
\hline $1 \mathrm{H}$ & 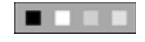 & Color change & .88 & .89 & 7 & $>.50$ \\
\hline $1 \mathrm{I}$ & $1+$ & Shape change & .91 & .90 & 7 & $>.50$ \\
\hline $1 \mathrm{~J}$ & $\bullet$ & Size change & .81 & .84 & 7 & $>.25$ \\
\hline
\end{tabular}

Note-Ranges of $A^{\prime}$ from .5 to 1.0 , where .5 corresponds to chance performance, and 1.0 corresponds to perfect performance. *With comparable amount of end point changes, rotation produced a larger disruption effect than length change did. 

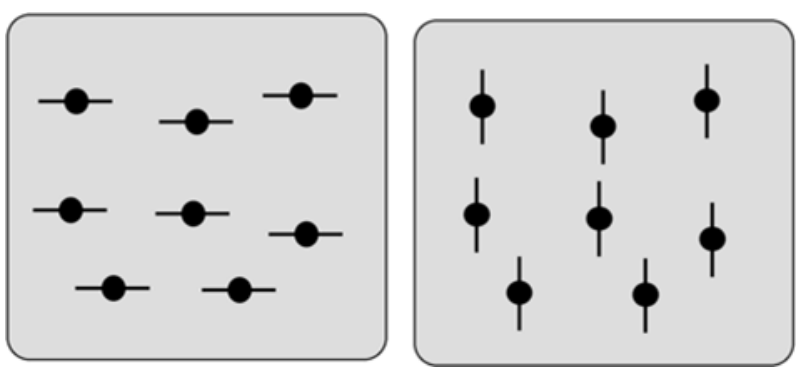

Figure 2. Change in the orientation of the long axis of an item leads to a change in perceptual grouping.

(Experiment 2B). Rotations of line stimuli orientation did not affect the location of each item within the grid, and the rotations did not change the shape of the chromatic links. Thus, the regular grid or the links provided constant information that was unaffected by line orientation change.

The constancy provided by the reference frames might help subjects ignore orientation changes. However, it is not obvious whether such constant, invariant cues are sufficient to reduce or remove the disruption effect. In fact, Experiment 1 showed that the presence of invariant information was not always sufficient. When dots were presented at the center of each line, rotating the line segments did not change the position of these salient dots (see Figure 1), yet the subjects could not ignore the orientation change. In fact, the invariant central dots failed to reduce the disruption. Moreover, unpublished data from our lab have revealed the same amount of disruption whether the line centers were marked or not. It is therefore of interest to find out whether other types of cues, such as the presence of a regular grid, might provide a constant reference frame that can reduce or eliminate the disruption effect.

\section{Method}

Experiment 2A. Sixteen observers were tested. Each observer performed 12 practice and 256 experimental trials. Two types of stimuli were tested: line arrays without an external background grid and line arrays appearing within a regularly spaced grid. As in Experiment 1 , we varied line orientation constancy from memory to probe displays and also varied the presence or absence of one location change. Ten observers were tested in a blocked design in which the presence or absence of the grid were tested in separate blocks, and six were tested in a mixed design. The results were pooled across these two groups, because the pattern of performance was unaffected by this design. Each display contained 12 items in heterogeneous orientations.

Experiment 2B. Eight observers performed 12 practice and 256 experimental trials. The design of this experiment was identical to that of Experiment 2A, except that we replaced the regularly spaced grid with a connect-the-dot chromatic grid that linked the centers of all the lines. The two types of displays (lines with or without chromatic links) were tested in separate blocks. The order of the blocks (ABBA or BAAB) was counterbalanced across observers. Each display contained eight items in homogeneous orientations.

\section{Results}

Figure 3 plots $A^{\prime}$ of location change detection as a function of item rotation and grouping cues. For Experiment 2A, a repeated measures analysis of variance (ANOVA) showed a significant main effect of line orientation change $[F(1,15)=12.11, p<.003]$ and a significant interaction between orientation change and the presence or absence of background grid $[F(1,15)=7.20$, $p<.017]$. The main effect of background grid was not significant $[F(1,15)=1.66, p>.20]$. Specifically, orientation change produced a significant disruption on change detection when the lines were presented without the background grid $[t(15)=4.45, p<.001]$. In contrast, orientation change had no effect on performance when the lines were presented on a regularly spaced background grid $[t(15)=1.39, p>.15]$. Thus, the presence of a background grid eliminated the disruption effect produced by line orientation change.

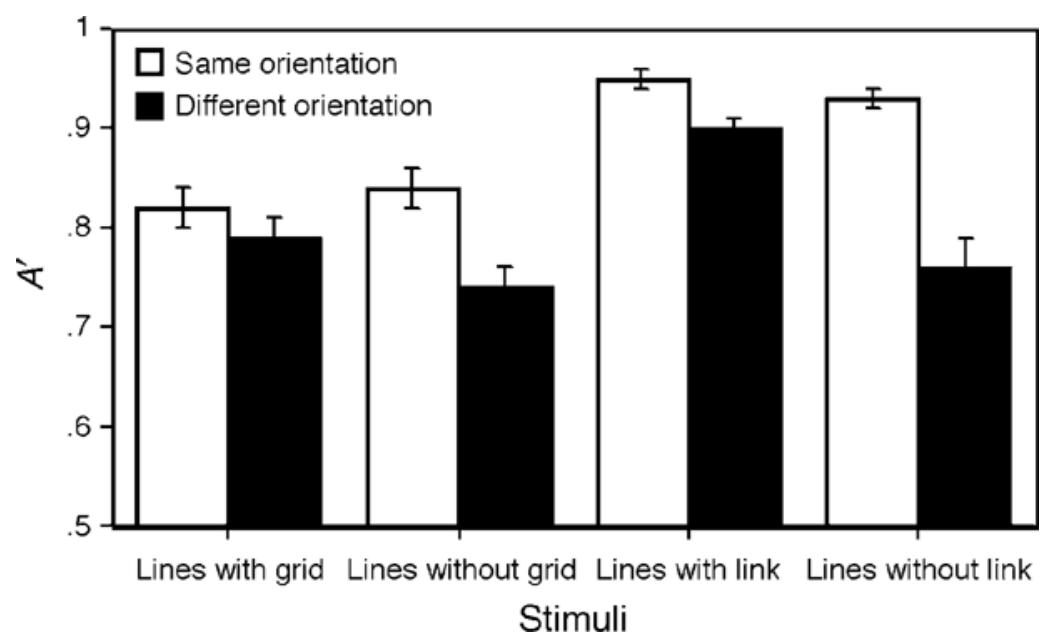

Figure 3. The results from Experiment 2. Error bars indicate standard error of between-subjects variation. 
For Experiment 2B, an ANOVA revealed a significant main effect of line orientation change $[F(1,7)=35.20$, $p<.001]$, a significant main effect of the presence or absence of chromatic links $[F(1,7)=30.60, p<.001]$, and a significant interaction between orientation change and chromatic links $[F(1,7)=16.49, p<.005]$. Pairwise $t$ tests showed that orientation change was disruptive both when the chromatic links were absent $(p<.001)$ and when they were present $(p<.002)$. However, the significant interaction indicated that the presence of a constant chromatic link attenuated the disruption effect of orientation change.

\section{EXPERIMENT 3}

In Experiment 1 (and also Experiments 1B-1E; see Table 1), we demonstrated that selective attention to one dimension of a stimulus (location) fails to filter out changes in another dimension (orientation) if there are multiple elongated items. This raises the question about whether the influence of orientation change is completely stimulus driven or whether it can be modulated by topdown attention. Studies directly addressing the relation between selective attention and perceptual grouping have shown that, on the one hand, the spread of attention is affected by perceptual grouping (Egly, Driver, \& Rafal, 1994; Lavie \& Driver, 1996; Vecera \& Farah, 1994), whereas on the other hand, top-down attention influences perceptual grouping (Peterson \& Gibson, 1994a, 1994b). Because perceptual grouping by line proximity appears to occur obligatorily, it is unclear whether it can be influenced by selective attention. In this experiment, we tested the effect of selectively attending to one group of objects over another group and measured the disruption of location change detection produced by orientation change of the attended versus the ignored group. Figure 4 (inset) shows an example of a display.

Each display contained two sets of items - red dots with an interposing red line segment and green dots with an interposing green line segment - randomly intermixed on a $10 \times 10$ invisible matrix. The subjects were instructed, in different blocks, to attend either to the red items or to the green items and to perform location change detection of the attended group of items. To test the effect of selective attention, we independently varied the probability of orientation change for the attended and the ignored sets. If subjects can effectively attend to the instructed group of items and if selective attention modulates which items enter perceptual grouping, then orientation changes of the attended group should have a larger effect than do orientation changes of the ignored group. However, if the influence of orientation changes is completely stimulus driven, we may not observe an effect of selective attention.

\section{Method}

Subjects. Seventeen observers participated in this experiment. Materials. Each display contained eight red items and eight green items, randomly intermixed on a $10 \times 10$ invisible matrix $\left(19^{\circ} \times 19^{\circ}\right)$. Each item was a filled $\operatorname{dot}\left(0.4^{\circ} \times 0.4^{\circ}\right)$ interposed with a line segment $\left(1.2^{\circ} \times 1.2^{\circ}\right)$ that had one of four possible orientations $\left(0^{\circ}, 45^{\circ}, 90^{\circ}\right.$, or $\left.135^{\circ}\right)$.

Procedure. The subjects were tested in 10 practice trials and 4 blocks of experimental trials containing 48 trials each. Before each block, they were instructed to attend either to the green or to the red



Figure 4. The results from Experiment 3. A sample display is shown in the inset of the figure. Red (shown as black items on this figure) and green (shown as white items on this figure) items were randomly intermixed on the display. Error bars indicate standard error of between-subjects variation. 
items and to perform a location change detection on the filled dots. Each trial started with a 400-msec fixation point, followed by a memory display (eight red items and eight green items). The memory display was presented for $400 \mathrm{msec}$ and then was erased. After a blank interval of $1 \mathrm{sec}$, a probe display was presented until a response was made. The probe display also contained eight red and eight green items. Half of the time one of the attended items changed its location to a previously blank location, and half of the time the attended items maintained their locations. The ignored items never changed locations. The subjects were asked to press a right key if they detected a location change and a left key otherwise. They were told to ignore the oriented lines and their orientation changes. The attended items changed their orientations on $50 \%$ of the trials. Furthermore, the ignored items also changed their orientations on $50 \%$ of the trials.

All the factors tested in this experiment were independently manipulated. Thus, the subjects were tested in a total of 192 experimental trials, which can be broken down as follows: 2 attended colors (red vs. green) $\times 2$ location changes (change vs. no change) $\times$ 2 attended items' orientations (change vs. no change) $\times 2$ ignored items' orientations (change vs. no change) $\times 12$ cases.

\section{Results}

We pooled data across blocks of attending to red and blocks of attending to green and measured $A^{\prime}$ separately for four conditions: both attended and ignored groups maintained their orientations (both same), ignored group changed orientations (ignored different), attended group changed orientations (attended different), and both groups changed orientations (both different). Figure 4 shows the results.

An ANOVA on attended group and ignored group revealed a significant main effect of attended group $[F(1,16)=5.78, p<.029]$, showing lowered $A^{\prime}$ for location change detection when the attended group of items changed orientations. The main effect of ignored group was not significant $[F(1,16)=1.10, p>.30]$, suggesting that performance was not disrupted by orientation changes in the ignored group. The interaction between attended group and ignored group was not significant $[F(1,16)=2.41, p>.14]$. Planned contrasts showed that orientation change in the attended group alone was sufficient to disrupt performance $[t(16)=2.47, p<.05]$. Nonetheless, orientation change in the ignored group alone produced a marginally significant reduction in performance $[t(16)=2.22, p<.081]$. This could be caused by attention leaking to the ignored items or by a stimulusdriven effect of obligatory grouping of every item on the display. Thus, even though this experiment did not allow us to completely rule out bottom-up effects of orientation change on location change detection, it provided clear evidence that such effects were significantly modulated by selective attention.

\section{EXPERIMENT 4}

Two factors jointly contribute to the effect of orientation change on location change detection. First, the spatial relationship among items is obligatorily encoded in the representation of multiple locations (Jiang et al., 2000). Second, when constructing the global configuration, subjects are unable to extract just the pattern formed by the dots. Instead, the imaginary contour formed by the elongated objects becomes part of the global pattern, which changes when the objects rotate. In Experiment 2, we tried to disrupt the representation of the global pattern by connecting the center of the dots or by presenting a regular grid that served as an external frame of reference to represent individual dots. This largely reduced the orientation change effect.

In this experiment, we further eliminated the need to encode the global configuration by informing the subjects about which single item might change location. ${ }^{1}$ The informative cue serves to narrow the focus of spatial attention to a single location, removing the necessity to represent all the items and their configuration. If global shape processing is partly responsible for the disruption effect, then no disruption from orientation is expected when subjects focus on a single item.

\section{Method}

Subjects. Six observers were tested.

Materials. Each display contained eight dots that were interposed by a short line segment (see Experiment 1 and Figure 1). The first display was presented for $150 \mathrm{msec}$ and was then erased. One second later, another display was presented until a response was made. The first display was presented briefly to eliminate eye movement.

Design. The subjects were tested in two conditions: diffuse attention and focal attention (Figure 5). In the diffuse attention condition, the first display contained eight black dots and the second display contained one black dot and seven white dots. The subjects were asked to decide whether or not the black dot on the second display was at one of the previous eight locations. Because they had to monitor all the dots on the first display, attention was diffuse, so the subjects would have needed to encode the individual locations and their global configuration. In the focal attention condition, the first display contained one black dot and seven white dots, as did the second display. The subjects were asked to decide whether the black dot on the second display was at the same location as the one on the first display. Attention was narrow because only a single location needed to be extracted and monitored from the first display, and thus there was no need to encode the global configuration. Figure 5 shows an example of the display.

The subjects were tested in 20 practice trials and 160 experimental trials. These included a factorial design of three factors: extent of spatial attention (diffuse vs. focal), location change (same vs. different), and orientation change (same vs. different). An orientation change meant that all items rotated by $90^{\circ}$, including the item(s) that the subjects were monitoring.

\section{Results and Discussion}

The results are shown in Figure 5. We calculated $A^{\prime}$ as well as mean response time (RT) from each subject's median RT (for correct trials only). An ANOVA on attention and orientation change showed that performance was better when the subjects monitored one location than when they monitored eight $[F(1,5)=39.59, p<$ $.001]$ and when the orientation of the irrelevant line segments stayed the same $[F(1,5)=12.81, p<.016]$. There was also a significant interaction between set size and orientation change $[F(1,5)=18.72, p<.008]$, suggesting that the effect of orientation change was modulated by how diffuse spatial attention was. Orientation change disrupted performance only when the subjects attended 

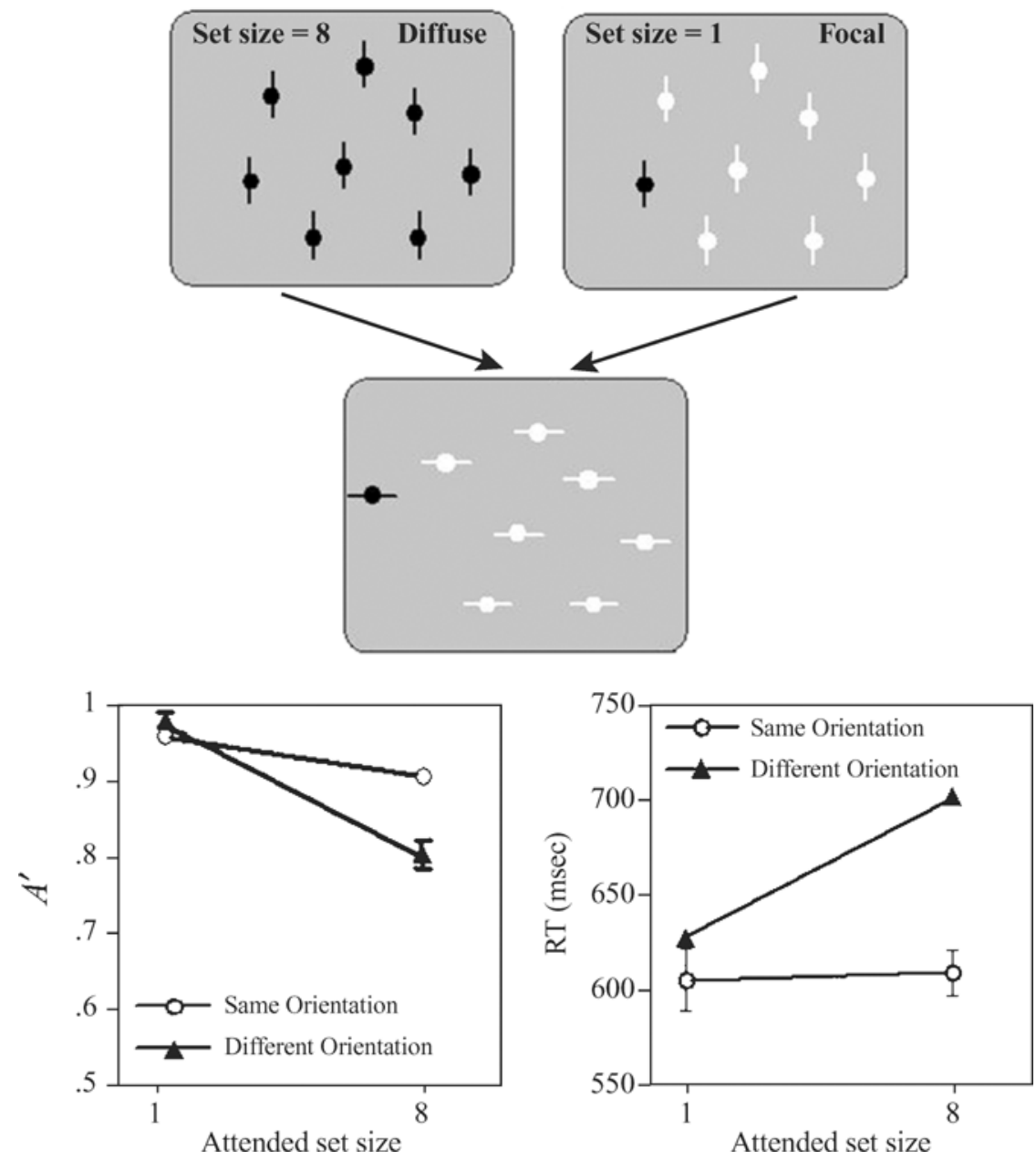

Figure 5. Sample displays and results from Experiment 4. The subjects monitored the locations of the black dots. Error bars indicate the standard error of the difference between same orientation and different orientation.

to all locations $[t(5)=4.34, p<.007]$ but not when they focused on one location $[t(5)=0.99, p>.35]$.

A similar pattern of results is seen in RTs. An ANOVA showed significant effects of set size $[F(1,5)=26.93, p<$ $.003]$ and orientation change $[F(1,5)=39.83, p<.001]$ and a significant interaction $[F(1,5)=9.44, p<.03]$. Orientation change lengthened RTs when the subjects monitored all locations $[t(5)=7.76, p<.001]$ but not when they focused on one location $[t(5)=1.32, p>.20]$.

\section{GENERAL DISCUSSION}

This study provides a compelling demonstration that the perceptual grouping of individual items can significantly affect change detection, even when subjects are instructed to ignore the grouping. When subjects had to focus on change detection of one dimension (the spatial location of elongated items), they were unable to ignore changes in another dimension (the orientations of the items). We believe that this is because changes in the ori- entations of elongated objects altered their global perceptual organization. This disruption can be reduced by an invariant frame of reference, such as a regularly spaced background grid (Experiment 2A), by actively using color feature cues to ignore the group of items that underwent orientation changes (Experiment 3), or by narrowing the focus of attention to a single item (Experiment 4). However, the presence of constant information was not always sufficient (Experiment 1), and the task demand to ignore orientation change of the attended set of items was not always effective.

The disruption of location change detection by orientation change is the outcome of two interrelated processes. First, when multiple locations need to be retained briefly, the visual system does not simply encode each individual spatial location in an egocentric or environmentbased frame of reference. Instead, the pattern formed by all the elements is obligatorily encoded and retained. A change in the global configuration of items severely disrupts change detection of individual locations (Jiang 
et al., 2000; Santa, 1977). Configural encoding is modulated by attention: Only items that are actively attended become part of the configuration (Jiang et al., 2000). As a result, changes to irrelevant locations are negligible (Experiments 3 and 4).

Perceptual grouping, which is related to configural processing, influences how a configuration may be formed. Thus, manipulations that might change the perceived configuration can potentially disrupt the representation of locations. Under many conditions, selective attention can override the potential disruption produced by irrelevant changes. For example, differences in color grouping from memory to probe displays were negligible during location change detection. This suggests that perceptual grouping by color does not influence the spatial configuration of items. The special case that cannot be disregarded is the orientation change in the long axis of elongated objects, even when the centers of these objects were clearly demarcated.

Configural encoding alone cannot explain the effects of orientation change. This is because the configuration formed by the dots remained the same across the two displays. The results can be accounted for only if the configuration included not just the center dots but also the long axes. Orientation change alone also fails to explain the disruption effect, because orientation change of a single item (Experiment 4), irrelevant items (Experiment 3 ), or items encoded within an environment-based frame of reference (Experiment 2A) did not influence change detection of locations. Such effects, therefore, must arise from the interaction among elongated objects in multi-element displays. That is, the relative spatial locations are no longer preserved when the long axes of these objects are rotated.

Although we did not test every kind of grouping change that might influence change detection of locations, the analysis above suggests that only grouping cues that influence the relative spatial location between items will have an effect. Grouping cues that affect similarity without changing perceived proximity, such as color grouping, do not influence location change detection. On the basis of this logic, the present paradigm provides a tool to quantify whether certain grouping cues influence the perception of spatial locations.

In conclusion, our study provides further support that items are represented in a relational manner in change detection (Jiang et al., 2000). Perceptual grouping between items can affect how each item is represented, even when the grouping is task irrelevant. Whether or not grouping defined by other kinds of visual features is also represented in change detection remains to be seen.

\section{REFERENCES}

Donaldson, W. (1993). Accuracy of $d^{\prime}$ and $A^{\prime}$ as estimates of sensitivity. Bulletin of the Psychonomic Society, 31, 271-274.

EgLY, R., DrIVER, J., \& RAFAL, R. (1994). Shifting visual attention between objects and locations: Evidence from normal and parietallesion patients. Journal of Experimental Psychology: General, 123, 161-177.

GRIER, J. B. (1971). Nonparametric indexes for sensitivity and bias: Computing formulas. Psychological Bulletin, 75, 424-429.

HunT, S. M. J. (1994). MacProbe: A Macintosh-based experimenter's workstation for the cognitive sciences. Behavior Research Methods, Instruments, \& Computers, 26, 345-351.

JiAnG, Y., Olson, I. R., \& CHUn, M. M. (2000). Organization of visual short-term memory. Journal of Experimental Psychology: Learning, Memory, \& Cognition, 26, 683-702.

LAVIE, N., \& Driver, J. (1996). On the spatial extent of attention in object-based visual selection. Perception \& Psychophysics, 58, 12381251.

LucK, S. J., \& Vogel, E. K. (1997, November). The capacity of visual working memory for features and conjunctions. Nature, 390, 279281.

Palmer, S. E. (1999). Vision science: Photons to phenomenology. Cambridge, MA: MIT Press.

PAshler, H. (1988). Familiarity and visual change detection. Perception \& Psychophysics, 44, 369-378.

Peterson, M. A., \& Gibson, B. S. (1994a). Must shape recognition follow figure-ground organization? An assumption in peril. Psychological Science, 5, 253-259.

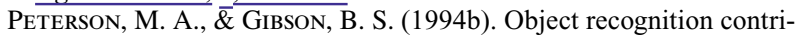 butions to figure-ground organization: Operations on outlines and subjective contours. Perception \& Psychophysics, 56, 551-564.

Phillips, W. A. (1974). On the distinction between sensory storage and short-term visual memory. Perception \& Psychophysics, 16, 283 290.

Rensink, R. A. (2002). Change detection. Annual Review of Psychology, 53, 245-277.

RENSINK, R. A., O'REgan, J. K., \& ClaRK, J. J. (1997). To see or not to see: The need for attention to perceive changes in scenes. Psychological Science, 8, 368-373.

SANTA, J. L. (1977). Spatial transformations of words and pictures. Journal of Experimental Psychology: Human Learning \& Memory, 3, 418-427.

Simons, D. J., \& Levin, D. T. (1997). Change blindness. Trends in Cognitive Sciences, 1, 261-267.

Tulving, E. (1974). Cue-dependent forgetting. American Scientist, 62, 74-82.

Vecera, S. P., \& Farah, M. J. (1994). Does visual attention select objects or locations? Journal of Experimental Psychology: General, 123, 146-160.

WoOdman, G. F., Vecera, S. P., \& Luck, S. J. (2003). Perceptual organization influences visual working memory. Psychonomic Bulletin \& $\underline{\text { Review, 10, }} \underline{\text { 80-87. }}$

\section{NOTE}

1. We thank Robert Melara for suggesting this manipulation.

(Manuscript received January 10, 2003; revision accepted for publication August 25, 2003.) 